\title{
Influence of Ammonium Zirconium Carbonate on Properties of Poly(vinyl alcohol)/Xylan Composite Films
}

\author{
Xiao-feng Chen, ${ }^{1}$ Jun-li Ren, ${ }^{1}$ and Ling Meng ${ }^{2}$ \\ ${ }^{1}$ State Key Laboratory of Pulp and Paper Engineering, South China University of Technology, Guangzhou 510640, China \\ ${ }^{2}$ Shanghai Haisum Chemical Technology Co., Ltd., Shanghai 200030, China \\ Correspondence should be addressed to Jun-li Ren; renjunli@scut.edu.cn
}

Received 17 March 2015; Revised 15 May 2015; Accepted 18 May 2015

Academic Editor: Ilaria Armentano

Copyright (c) 2015 Xiao-feng Chen et al. This is an open access article distributed under the Creative Commons Attribution License, which permits unrestricted use, distribution, and reproduction in any medium, provided the original work is properly cited.

\begin{abstract}
A series of composite films were prepared using poly(vinyl alcohol) (PVA) and xylan as degradable raw materials under the addition of glycerol and ammonium zirconium carbonate (AZC). The influences of AZC on the mechanical properties, waterresisting properties, thermal stability, solubility $(S)$, and water vapor permeability of PVA/xylan composite films were comparatively discussed. The results showed that AZC had a positive impact on improving the water resistance and mechanical properties of composite films especially for elongation at break (EAB). With increasing the AZC amounts from $0 \%$ to $15 \%$, EAB increased rapidly from $18.5 \%$ to $218.0 \%$, and the $S$ values decrease from $11.64 \%$ to $8.64 \%$. When the AZC amount reached $15 \%$, the tensile strength still performed well $(22.10 \mathrm{MPa})$. The great compatibility of components in composite films was also observed. Moreover, the addition of AZC had great influences on the thermal stability of composite films and the degradation in soil.
\end{abstract}

\section{Introduction}

Because of the availability at the relatively low cost and the good mechanical performance, petrochemical-based plastics have been widely used in the food packaging industry and the agricultural field, while they are mostly nonbiodegradable [1]. With the environmental issue being more and more concerned and rapidly depleting petroleum reserves, there has been an increased interest in plastics based on renewable and degradable resources, including the natural resource such as starch, cellulose, and chitosan.

Hemicelluloses, as a promising renewable raw material source, are considered to be the second most abundant polysaccharide in nature. And xylan is the most common hemicelluloses of the plant kingdom [2]. Even though there are various reports on the films made of natural biopolymers, studies on xylan films have been limited because the pure xylan performed a poor film-forming ability [3]. An efficient approach to improve the film-forming property of xylan was to combine with compatible polysaccharides, proteins, and some other polymers [4]. The film-forming ability of xylan at various mixtures of chitosan was studied [3], and the result exhibited a better film-forming property when the amount of chitosan increased, while the crystallinity of the films decreased. Composite films were prepared by casting the solutions of cotton stalk xylan and birchwood xylan mixed with lignin, respectively. It was found that the mechanical properties and water vapor transfer rates of films were improved when xylan amounts increased from $8 \%$ to $14 \%$ [5]. In addition, cellulose nanofibers (CNFs) were also incorporated into xylan films to enhance the mechanical properties of xylan-based films [6]. The addition of $5 \mathrm{wt} \%$ CNF could increase the tensile stress and Young's modulus of composite film by 30 and $80 \%$, respectively. And the addition of $5 \mathrm{wt} \% \mathrm{CNF}$ achieved the highest tensile stress (39.5 MPa) and the highest Young modulus (3404 MPa). Poly(vinyl alcohol)/xylan composite films were fabricated with glycerol, urea, and 1,2,3,4-butane tetracarboxylic acid as a new plasticizer or a cross-linking agent $[7,8]$. Poly(vinyl alcohol) (PVA) could remarkably improve the mechanical properties of xylan films. The addition of urea in PVA/xylan composite films also improved the water resistance property. As a result, the contact angle reached $114.68^{\circ}$. Another method to improve the film-forming ability of xylan was to modify xylan. The films prepared from carboxymethyl xylan (CMX) were examined for mechanical properties and their 
suitability as a film for packaging applications [9]. Increasing the degree of substitution (DS) of CMX films resulted in an increase in elongation at break (EAB) and a decrease in tensile strength (TS) and Young's modulus. The DS also affected the barrier properties of the films. CMX films with higher DS showed improved (reduced) oxygen permeability (OP). Moreover, the water vapor permeability (WVP) increased with increasing DS. Xylan was modified with propylene oxide to prepare water-soluble hydroxypropyl xylan (HPX). Then the HPX-films were prepared and the mechanical properties were investigated [10]. Results showed the ultimate strength of HPX-films were almost 6-8 times higher than the corresponding hydroxypropyl cellulose. Before intercalating the montmorillonite (MMT) nanoplatelets to prepared biopolymer-clay composite films, the quaternized xylantype hemicelluloses were synthesized by the etherification of quaternary ammonium salt reagents [11]. Acetylated arabinoxylans (AX) films with different arabinosyl substitution level were prepared [12]. In this test, the effects of arabinosyl substitution on properties were evaluated. A higher arabinosyl substitution would improve the flexibility and decrease the glass transition temperatures $\left(T_{g}\right)$ of the films. The strain at break (22\%) and the ultimate strength up to $60 \mathrm{MPa}$ showed good mechanical properties, which would be suitable for the packaging application.

PVA is the sole vinyl polymer that can be used as the carbon source and energy for the bacteria. It has a good biodegradability and an excellent film-forming ability. PVA is one polymer used as a component for preparing composite film by blending with some natural polymers. Starch/PVA films were prepared by adding four different cross-linking agents including borax, formaldehyde, epichlorohydrin, and $\mathrm{ZnO}$ [13]. Results showed that the borax cross-linked film showed the highest tensile strength and the epichlorohydrin cross-linked film revealed the highest flexibility. Compared to the uncross-linked films, the values of TS and EAB increased up by $160 \%$ and $80 \%$, respectively. All the crosslinked films displayed lower moisture absorption than the uncross-linked films. Cellulose/PVA biocomposite films were prepared by dissolving in the ionic liquid 1-n-butyl-3methylimidazolium chloride. The films showed the good optical transparency and thermal stabilities properties. And compared to cellulose films, the strain at break of composite films increased up from $24.7 \%$ to $30.6 \%$ [14]. Moreover, chitin and chitosan were also used to fabricate PVA composite films $[15,16]$. The prepared $\alpha$-chitin nanofibers-PVA composite films showed high tensile strength (127 Mpa) and modulus (5.70 GPa), while EAB decreased to $4.40 \%$. And the $\mathrm{PVA} /$ chitosan hydrogel thin films loaded silver nanoparticles revealed good mechanical and biocompatible properties. However, relatively fewer researches have been focused on PVA/xylan composite films $[7,8]$.

To improve the elasticity of films, plasticizers are usually added into polymer solution. It has been reported that nontoxic ammonium zirconium carbonate (AZC) can cross-link hydroxyl or carboxyl groups in water [17]. The mechanism and kinetics of AZC self-cross-linking and AZC-starch cocross-linking in aqueous solutions were studied [18]. The result showed that the gel strength exhibited a power law scaling with polymer concentrations. Composite films were prepared via hydrogen bonding of the hydroxyl groups of galactoglucomannan (GGM) and AZC [19]. Result showed that AZC had a positive impact on decreasing the water permeation property of the films. AZC could be used as a cross-linking agent in xylan-based films to decrease their water solubilities [20].

The objective of the present study was to prepare AZCloaded PVA/xylan composite films, and the influences of the AZC amount on the properties of composite films were comparatively investigated such as mechanical properties, water-resisting properties, thermal stability, solubility $(S)$ and water vapor permeability, and the degradability in soil. The compatibility of composite films was also discussed. It would develop the fundamental investigation of PVA/xylan composite films with the inorganic additives and have promising applications in agriculture and industry. The novelty of the research was the choice of the inorganic additive (AZC). There were few investigations about the influence of inorganic additive on properties of PVA/xylan composite films. Compared to the PVA/xylan composite films fabricated with 1,2,3,4-butane tetracarboxylic acid [8], the PVA/xylan composite films fabricated with AZC showed similar TS and were several times better in EAB. Moreover, the PVA/xylan composite films fabricated with AZC exhibited better wet strength, which was reflected in the lower solubility. It indicated that the inorganic additive AZC had better effect on some properties of PVA/xylan composite films than some organic additives.

\section{Experimental}

2.1. Materials. Beech wood xylan $\left(M_{w}\right.$ of $\left.13,000 \mathrm{~g} / \mathrm{mol}\right)$ and PVA $\left(M_{w}\right.$ of $14,600 \sim 18,600 \mathrm{~g} / \mathrm{mol}$ and degree of hydrolysis of 99\%) were purchased from Sigma Aldrich (USA) and were used without further purification. AZC was supplied by Adamas-beta Reagent Company Limited. Glycerol and anhydrous $\mathrm{CaCl}_{2}$ and $\mathrm{NaCl}$ were of analytical-reagent grade and were obtained from Guangzhou Chemical Reagent Factory (Guangzhou, China). Ultrapure water was used in all experiments.

2.2. Preparation of PVA/Xylan Composite Films Containing AZC. The composite films were obtained by the solution casting method at a $3: 1$ weight ratio of PVA and xylan. $1.5 \mathrm{~g}$ PVA was dissolved in $90 \mathrm{~mL}$ ultrapure water in a $95^{\circ} \mathrm{C}$ oil bath for $1 \mathrm{~h}$ under stirring. After a PVA homogeneous solution being formed, $0.5 \mathrm{~g}$ xylan and $10 \%$ (wt $\%$, based on the weight of PVA and xylan) glycerol dissolved in $5 \mathrm{~mL}$ ultrapure water were added to the PVA solution at $80^{\circ} \mathrm{C}$. The mixture was stirred for $1 \mathrm{~h}$. Then different amounts of AZC in the range of $0 \%-15 \%$ (wt\%, based on the weight of PVA and xylan) were dissolved in $5 \mathrm{~mL}$ ultrapure water and added into the mixture. The final solution was kept at $80^{\circ} \mathrm{C}$ for $5 \mathrm{~h}$. Finally, the solution was filtered and the filtrate was poured into a teflon mould. Water was evaporated slowly in a ventilated oven at $40^{\circ} \mathrm{C}$ overnight. The films were then peeled off and reserved at $23^{\circ} \mathrm{C}$ with $50 \%$ relative humidity $(\mathrm{RH})$ at least $48 \mathrm{~h}$ prior to all measurements. 
2.3. Film Thickness. The film thickness was measured by employing a Lorentzen \& Wettre thickness gauge (Sweden). Measurements were performed at 5 different locations on films, and the average value was used in all of the following calculations.

2.4. Mechanical Properties. Determination of TS and EAB of films was performed with an Instron Universal Testing Machine (Instron Universal Test Machine Model 5565) with a $100 \mathrm{~N}$ load cell. The samples were cut in the rectangular specimens with a width of $15 \mathrm{~mm}$ and length of $150 \mathrm{~mm}$ by a paper cutter (FQQZD15, Sichuan, China), and five replicate specimens were measured from each film type. The test was operated at a tensile speed of $20 \mathrm{~mm} / \mathrm{min}$ with initial grip length of $30 \mathrm{~mm}$. All the measurements were determined in a constant temperature and humidity chamber $\left(23^{\circ} \mathrm{C} / 50 \%\right.$ $\mathrm{RH})$ and the mechanical tensile data were averaged over five specimens.

2.5. Contact Angle of Water Measurement. The hydrophobicity of films was evaluated using an OCA40 contact angle system apparatus (Dataphysics, Filderstadt, Germany) by measuring the contact angles of a water droplet $(5 \mu \mathrm{L}) 5 \mathrm{~s}$ after the water droplet landed on the films. Contact angles were determined at five different positions on each film.

2.6. Solubility. The films predried were cut into $2.5 \times 2.5 \mathrm{~cm}^{2}$, put into $50 \mathrm{~mL}$ of ultrapure water, and swollen for $24 \mathrm{~h}$ at room temperature. After this process, the swollen films were dried again at $105^{\circ} \mathrm{C}$ to the constant weight. $S$ was calculated by

$$
S=\frac{\left(m_{0}-m_{2}\right)}{m_{0}}
$$

where $m_{0}$ is the predry weight of PVA/xylan composite film (g); $m_{2}$ is the first dry weight of the swollen PVA/xylan composite film (g).

2.7. Water Vapor Permeability. The WVP was gravimetrically determined according to a procedure [21]. Five film samples were clamped on the top of polyethylene bottles containing $3 \mathrm{~g}$ anhydrous $\mathrm{CaCl}_{2}$ to maintain $0 \% \mathrm{RH}$ inside the bottles, followed by sealing the film over the edge of bottles with vaseline. Then the bottles were put into a dryer, in which $500 \mathrm{~mL}$ of saturated $\mathrm{NaCl}$ solution was placed to maintain a constant environment $\left(23^{\circ} \mathrm{C} / 75 \% \mathrm{RH}\right)$. The bottle was weighed every $2 \mathrm{~h}$, and WVP value was calculated according to

$$
\mathrm{WVP}=\frac{(\Delta m \times L)}{A \times t \times \Delta p},
$$

where WVP was the water vapor permeability $\left(\mathrm{g} \cdot \mathrm{m}^{-1} \cdot \mathrm{s}^{-1} \cdot \mathrm{Pa}^{-1}\right) . \Delta \mathrm{m} / \mathrm{t}$ was the gain rate of the bottle $\left(\mathrm{g} \cdot \mathrm{s}^{-1}\right)$. $A$ was the film area exposed to moisture transfer $\left(\mathrm{m}^{2}\right) . L$ was the mean thickness of the film (m). $\Delta p$ was the water vapor pressure difference $(\mathrm{Pa})$ between the two sides of the film $[22$, 23]. All samples were tested in triplicate to reduce the errors.
2.8. Characterization. Film samples were cut into pieces $(10 \mathrm{~mm} \times 10 \mathrm{~mm})$ and dried in the infrared drying oven. FTIR analysis was performed using a Fourier transform spectrophotometer (Nicolet 750, Florida, USA) appended to Attenuated Total Reflectance technique. A total of 32 scans were accumulated in transmission mode with a resolution of $4 \mathrm{~cm}^{-1}$. The spectrum was obtained from a range of $4000 \mathrm{~cm}^{-1}$ to $400 \mathrm{~cm}^{-1}$.

Thermal analysis (TGA) was carried out using thermogravimetric analysis on a simultaneous thermal analyzer (TGA Q500, TA Instruments, New Castle, USA). Specimens weighing approximately $9 \sim 11 \mathrm{mg}$ were heated from room temperature to $700^{\circ} \mathrm{C}$ at a heating rate of $10^{\circ} \mathrm{C} / \mathrm{min}$ in a nitrogen atmosphere. The apparatus was flushed with nitrogen at a flow rate of $20 \mathrm{~mL} / \mathrm{min}$.

The surface and cross-section of the samples were investigated using a scanning electron microscope analysis (SEM, Hitachi s-4300). In the test, $15 \mathrm{kv}$ acceleration voltage was used for the samples. The specimens were fixed on a metal stub and coated with gold using an Agar HR sputter coater.

2.9. The Degree of Degradation in Soil. The degree of degradation of the films was determined by the soil burial test. Selected film samples were cut into pieces $(30 \mathrm{~mm} \times 60 \mathrm{~mm})$ and then buried at the depth of $10 \mathrm{~cm}$ from the surface of the soil. A piece of gauze was covered on the film samples in order to find out the samples conveniently. The remaining samples were cleaned quickly and dried to a constant weight at $105^{\circ} \mathrm{C}$ every 10 days. The test would last for 60 days, which made the degree of degradation reach a roughly stable value. 60 -day test would try to make the xylan degrade completely. The degree of degradation was determined by

$$
\text { The degree of degradation }=\frac{\left(G_{1}-G_{2}\right)}{G_{1}} \times 100 \% \text {, }
$$

where $G_{1}$ is the weight of PVA/xylan composite film samples before burial (g); $G_{2}$ is the dry weight of the samples after burial for certain days $(\mathrm{g})$.

\section{Results and Discussion}

3.1. Mechanical Performance. PVA/xylan composite films have a range of thickness values from $50 \mu \mathrm{m}$ to $70 \mu \mathrm{m}$. Figure 1 illustrates the effect of the AZC amount on TS and EAB of the PVA/xylan composite films. Clearly, the addition of AZC led to a significant improvement in EAB of composite films. EAB increased rapidly from approximately $18.5 \%$ to $218.0 \%$ with increasing the amount of the AZC from $0 \%$ to $15 \%$. Obviously, AZC had a positive impact on the flexibilities of the composite films. This indicated that AZC mainly played a role as a plasticizer, which formed fewer covalent linkages with polymers in the test. AZC was loaded among the PVA and xylan chains which increased the interstitial volume of the material or the macromolecular mobility of the polymers. The PVA-xylan interactions decreased owing to the increase of plasticizer-macromolecular interactions. In view of the dominating plastification of AZC, it also had a certain impact on TS of composite films. When the amounts of AZC 


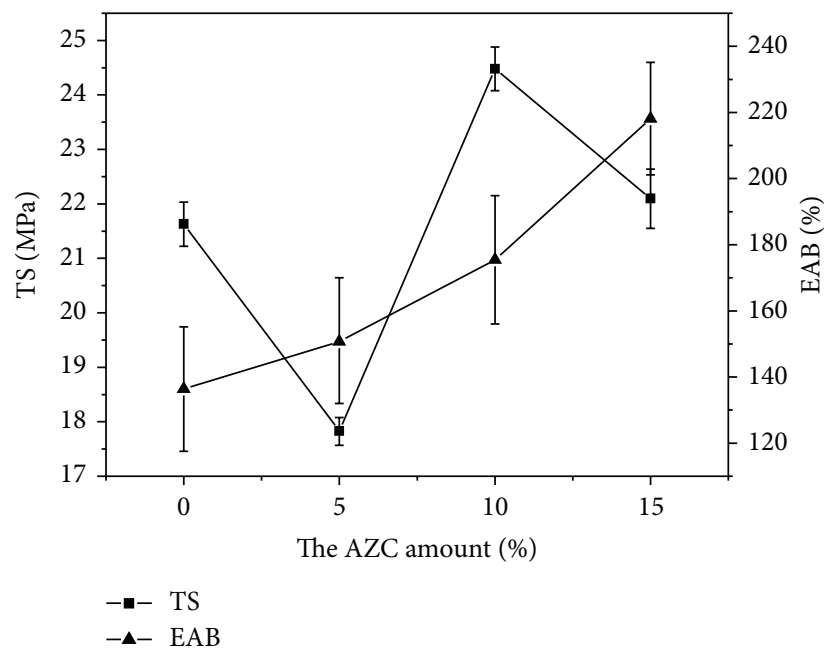

FIGURE 1: The effect of the AZC amount on TS and EAB of the PVA/xylan composite films.

TABLE 1: The effect of the AZC amount on $S$ and WVP of PVA/xylan composite films.

\begin{tabular}{lcc}
\hline Sample & WVP $\left(10^{-8} \mathrm{~g} \cdot \mathrm{m}^{-1} \cdot \mathrm{s}^{-1} \cdot \mathrm{Pa}^{-1}\right)$ & $S(\%)$ \\
\hline PVA & $4.2 \pm 0.3$ & $0.61 \pm 0.08$ \\
PVA-xylan & $3.2 \pm 0.2$ & $18.28 \pm 1.45$ \\
PVA-xylan-5\% AZC & $4.4 \pm 0.5$ & $11.64 \pm 0.98$ \\
PVA-xylan-10\% AZC & $3.7 \pm 0.5$ & $10.66 \pm 0.64$ \\
PVA-xylan-15\% AZC & $4.2 \pm 0.2$ & $8.64 \pm 1.12$ \\
\hline
\end{tabular}

increased from $5 \%$ to $15 \%$, TS firstly increased and then decreased. When the amount of AZC was $10 \%$, TS reached the highest values $(24.48 \mathrm{MPa})$. And the values of TS and EAB reached $22.10 \mathrm{MPa}$ and $218.12 \%$, respectively, as the amount of AZC was $15 \%$. Thus the strength of films performed well in the AZC amount range of $10 \%-15 \%$.

3.2. Contact Angle of Water Measurement. AZC had been used in producing water-resistant corrugated board [24]. In this study, AZC was employed to improve PVA/xylan composite films as a water-resistant agent by establishing hydrogen-bonds with the polymers. Contact angle of water drop is an indication of the hydrophobic properties or wettability of the film surface [25]. The effect of the AZC amount on the contact angle of water of the PVA/xylan composite films is illustrated in Figure 2. Clearly, AZC enhanced the hydrophobic characteristic of the composite films to some extent. Increasing the AZC amounts in hydrophilic composites resulted in an increment of the contact angle. Compared with the film without AZC, contact angle of the composite film containing 15\% AZC was increased by $50 \%$ approximately. This was ascribed to the decreased hydrophilic groups by forming hydrogen-bonds among components.

3.3. Solubility. Table 1 shows the effect of the AZC amount on the solubility of PVA/xylan composite films. The solubility of the composite films decreased with the increase of the AZC

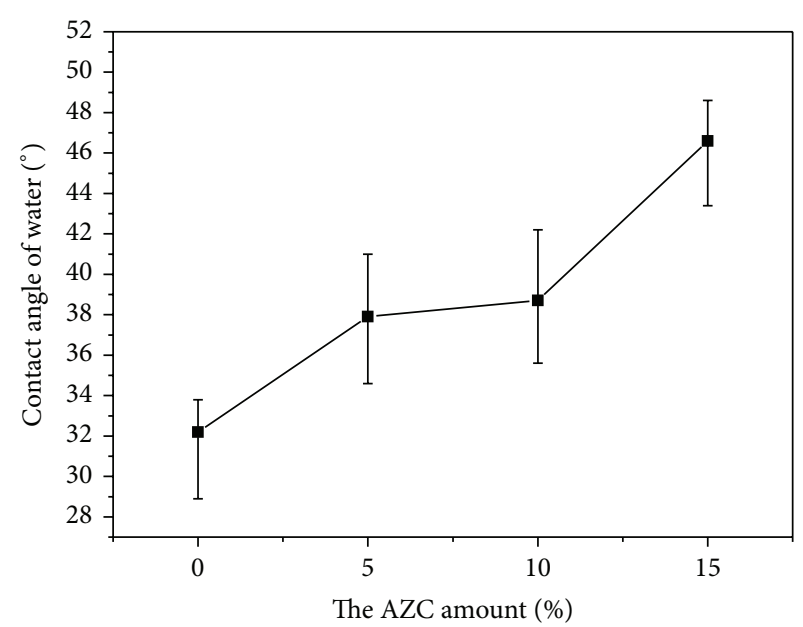

FIGURE 2: The effect of AZC amount on the contact angle of water of the PVA/xylan composite films.

amount. This could be explained by the increase of hydrophobic groups, which was due to the reaction of hydroxyl groups of PVA and xylan with AZC. This confirmed that AZC had in part played a role as a water-resistant agent again. A small number of hydrogen-bonds had larger effect than the introduction of hydrophilic groups. The solubility values $(8.64 \%-11.64 \%)$ were lower than that $(27.01 \%)$ obtained from PVA/xylan film with urea [7].

3.4. Water Vapor Permeability. The WVP values of PVA/xylan composite films obtained by the addition of different AZC amount are shown in Table 1. PVA/xylan film without AZC had the lowest WVP value $\left(3.2 \times 10^{-8} \mathrm{~g} \cdot \mathrm{m}^{-1} \cdot \mathrm{s}^{-1} \cdot \mathrm{Pa}^{-1}\right)$, which also was lower than that of PVA film. The obtained results confirmed that AZC had little impact on the WVP, which was due to few covalent linkages formation between polymers and AZC. The formed hydrogen-bonds were still not enough to improve the water vapor permeability significantly. Therefore, these results from mechanical properties, WVP, and contact angle measurement indicated that AZC played the key role as a plasticizer and had a certain influence on improving the hydrophobicity of composite films.

3.5. FTIR Analysis. FTIR spectra of the AZC-loaded PVA/xylan composite films are illustrated in Figure 3. The broad band ranging between 3,600 and $3,000 \mathrm{~cm}^{-1}$ is attributed to $\mathrm{O}$ $\mathrm{H}$ stretching vibration. It showed a decrease in the intensity of the $\mathrm{O}-\mathrm{H}$ band with increasing the amount of AZC. The $\mathrm{O}-\mathrm{H}$ group is hydrophilic. This intensity change suggested that the composite films became more hydrophobic under the function of AZC, which was consistent with the result of contact angle of water measurement.

3.6. Thermogravimetric Analysis. The thermal stabilities of PVA film and PVA/xylan composite films with and without AZC were assessed by TGA as shown in Figure 4. From the degradable curves, the thermogravimetric curves of the pure 


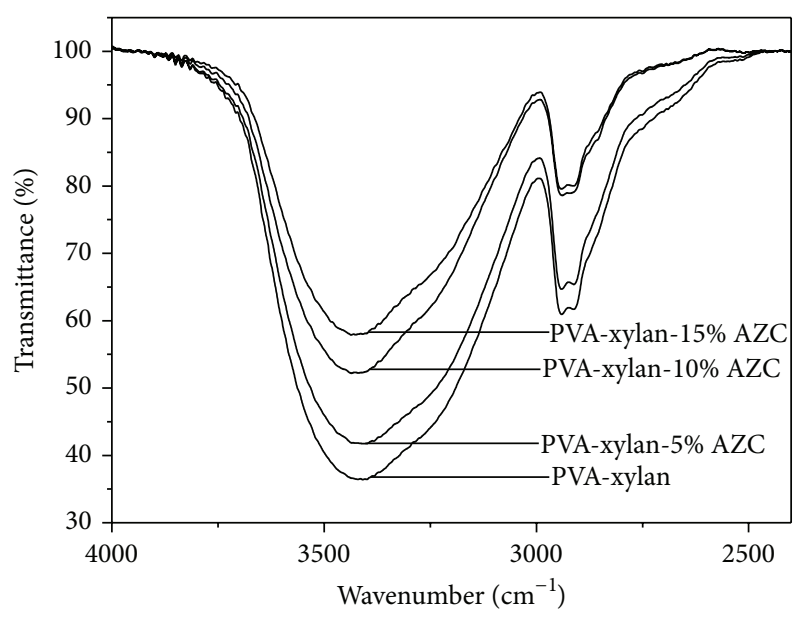

FIGURE 3: FTIR spectra of the AZC-loaded PVA/xylan composite films.

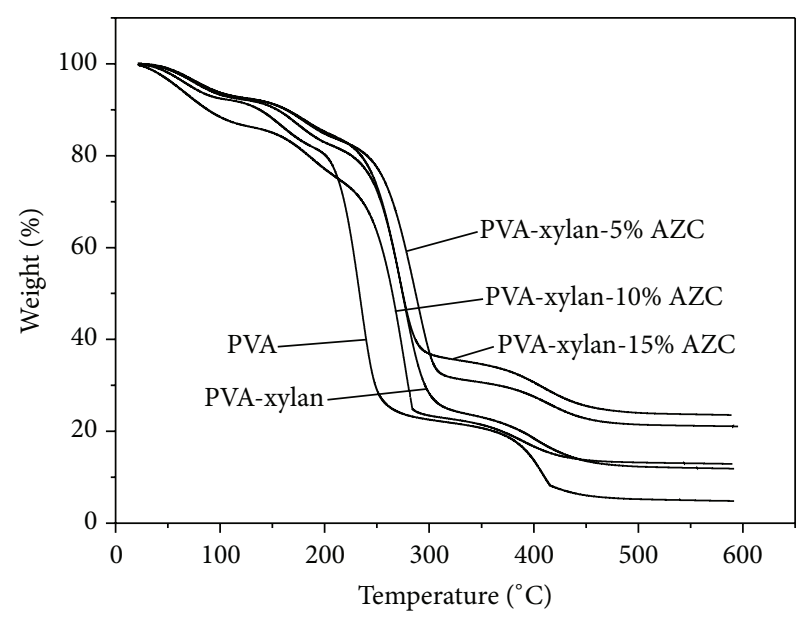

FIgURE 4: Thermogravimetric analysis of PVA film and PVA/xylan composite films with and without AZC.

PVA films were divided into the following three stages: $30-$ $210,210-250$, and $250-415^{\circ} \mathrm{C}$. The mass loss under $210^{\circ} \mathrm{C}$ was due to the loss of free water and bound water, the region about $210-250^{\circ} \mathrm{C}$ was attributed to the degradation of PVA chains, and the carbonation of the copolymer matrix occurred after $250^{\circ} \mathrm{C}$. Compared with the pure PVA films, the thermograms curves of the PVA/xylan composite films showed two main separate degradation phases: the first phase region around $240-310^{\circ} \mathrm{C}$ was due to the degradation of PVA and xylan side chains and the second phase region around $360-475^{\circ} \mathrm{C}$ was attributed to the cleavage of $\mathrm{C}-\mathrm{C}$ backbone in polymers matrix. For obtaining the same $50 \%$ residual weight of the total amounts, the decomposition temperature of PVA film occurred at $233^{\circ} \mathrm{C}$, but the composite films beyond $260^{\circ} \mathrm{C}$, even $287^{\circ} \mathrm{C}$ ( $5 \%$ AZC amount). The addition of AZC had significant influences on the degradation of PVA/xylan composite films. The AZC added PVA/xylan films had lower weight loss than PVA/xylan films after $450^{\circ} \mathrm{C}$, which indicated that the addition of AZC enhanced the thermal stabilities of PVA/xylan composite films. Moreover, the $15 \%$ amount of AZC added PVA/xylan films achieved the highest thermostability. The TGA results also implied an increase in the hydrophobicity of films after the addition of AZC, as showed by the lower weight loss at $100^{\circ} \mathrm{C}$, assigned to the water release, and compared to the PVA/xylan composite film without AZC [26].

3.7. Surface and Cross-Section Morphologies. Figure 5 displays the surface and cross-section morphologies of PVA/ xylan composite films with and without AZC. The SEM image of PVA/xylan composite film without AZC showed smooth surfaces, indicating the great compatibility and the favorable interaction between PVA and xylan. However, composite films with $10 \%$ and $15 \%$ AZC had smoother surfaces than films with the 5\% AZC, which implied that increasing AZC amounts could improve the compatibility among components in composite films. It was due to more hydrogenbonds formed at the function of AZC. Many particles in the cross-section were also observed clearly when the amount of AZC was $5 \%$, while the number of the particles became less after increasing the amount of AZC to $15 \%$. In consequence, an appropriate amount of AZC shows a positive effect on improving the compatibility among different components in the films.

3.8. The Degree of Degradation Analysis. The degradation of films in the soil as a function of time is presented in Figure 6. After the soil burial for 60 days, the PVA film had a degree of degradation which was about $10 \%$ but the composite films reached about $30 \%$. It could be explained that PVA can be efficiently degraded only in the presence of relatively uncommon microorganisms in natural environments [27]. Moreover, xylan could be more susceptible to microbial attack so the composite films had the relatively high degradation degree. The composite films without AZC had a higher degree of degradation (32\%) than composite films containing $10 \%$ AZC (27\%). This was ascribed to the difference in the relative content of xylan in composite films and the inorganic substance (AZC). Another possible reason was that the formed hydrogen-bonds of AZC with PVA and xylan reduced the degradation degree in soil caused by the microbial attack.

\section{Conclusions}

AZC was employed to improve the properties of PVA/xylan composite films obtained by casting method. Results showed that AZC had a positive impact on the mechanical property and the water resistance. When the amount of AZC was $15 \%$, TS and $\mathrm{EAB}$ of composite films reached $22.10 \mathrm{MPa}$ and $218.12 \%$, respectively. The results of the contact angle measurement and the FTIR characterization demonstrated that AZC could improve the hydrophobicity of the composite films, which decreased the solubility simultaneously. The favorable compatibility of composite films was confirmed by SEM. Furthermore, the composite films containing AZC showed the high thermal stability and the great degradability 

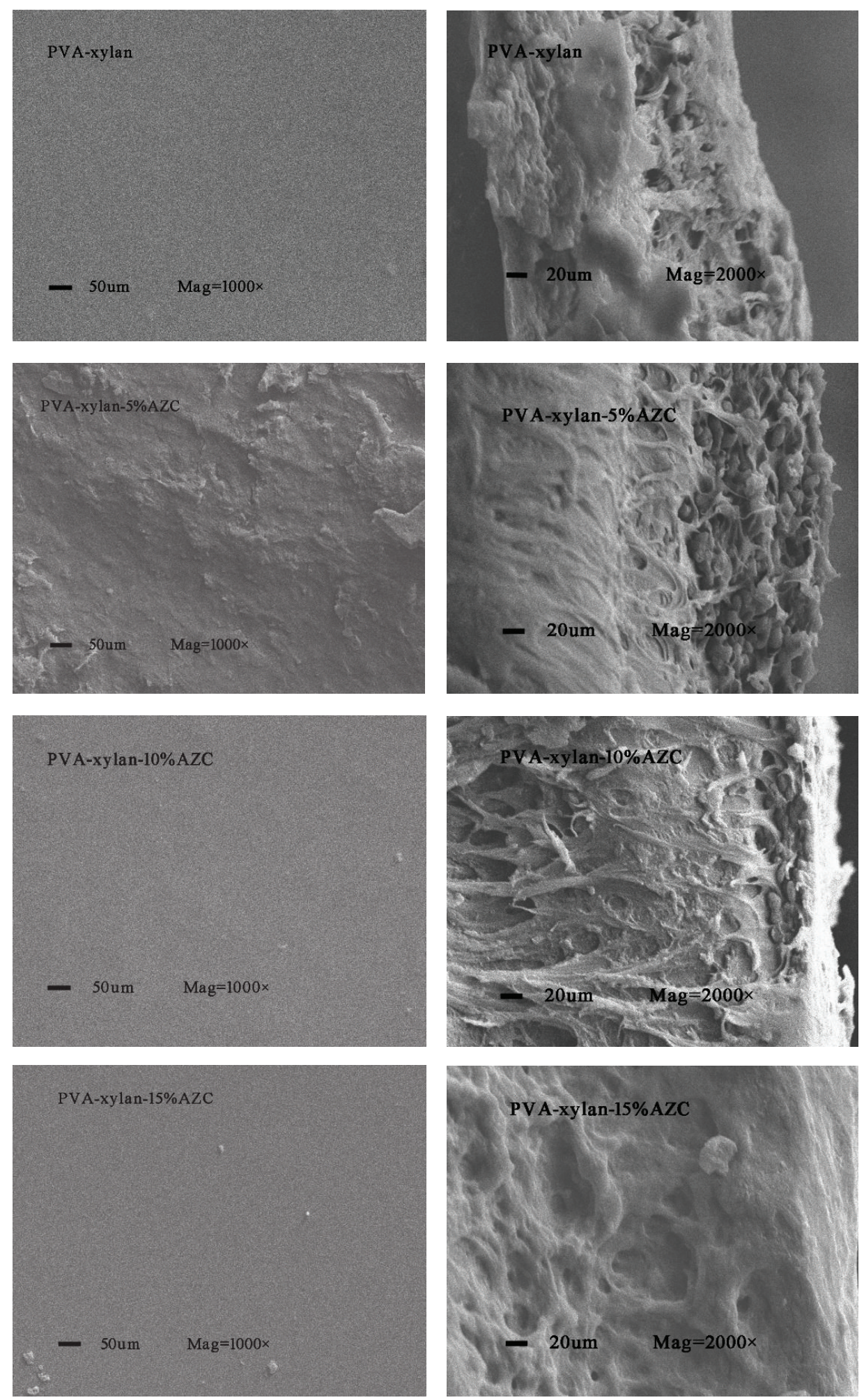

FIGURE 5: The surface $(\times 1,000$ magnification $)$ and cross-section $(\times 2,000$ magnification $)$ morphologies of PVA/xylan composite films with and without AZC. 


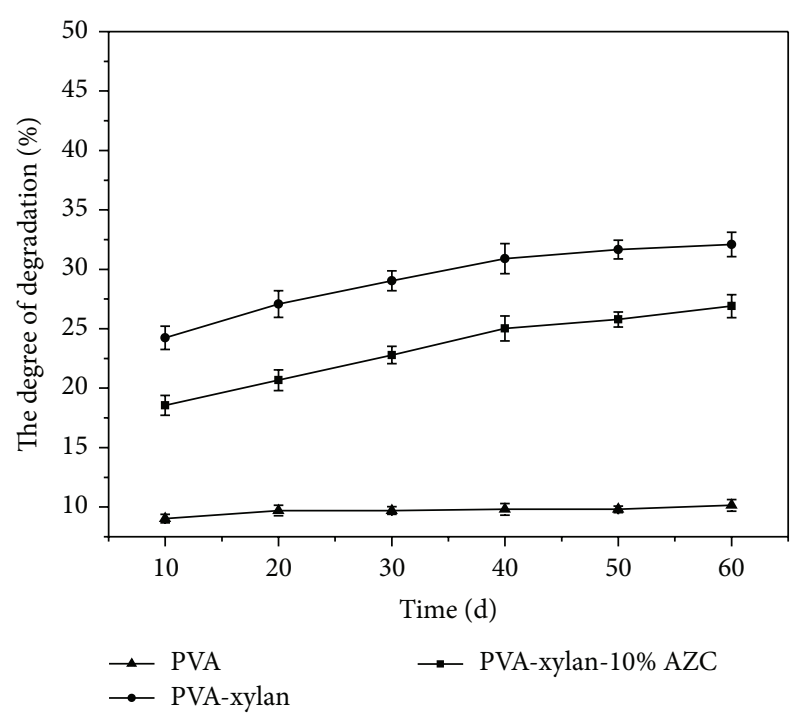

FIGURE 6: The degradation degree of PVA/xylan composite films in the soil as a function of time.

in soil. These results suggest that the composite films could be applied as degradable films in food packaging industry and agricultural field.

\section{Conflict of Interests}

The authors declare that there is no conflict of interests regarding the publication of this paper.

\section{Acknowledgments}

This work was supported by the grants from National Natural Science Foundation of China (no. 21406080), the Author of National Excellent Doctoral Dissertation of China (201169), and the Fundamental Research Funds for the Central Universities (2014ZG0003), SCUT.

\section{References}

[1] V. Siracusa, P. Rocculi, S. Romani, and M. D. Rosa, "Biodegradable polymers for food packaging: a review," Trends in Food Science \& Technology, vol. 19, no. 12, pp. 634-643, 2008.

[2] A. Ebringerová and T. Heinze, "Xylan and xylan derivatives biopolymers with valuable properties, 1: Naturally occurring xylans structures, isolation procedures and properties," Macromolecular Rapid Communications, vol. 21, no. 9, pp. 542-556, 2000.

[3] I. Gabrielii, P. Gatenholm, W. G. Glasser, R. K. Jain, and L. Kenne, "Separation, characterization and hydrogel-formation of hemicellulose from aspen wood," Carbohydrate Polymers, vol. 43, no. 4, pp. 367-374, 2000.

[4] B. Ş. Kayserilioğlu, U. Bakir, L. Yilmaz, and N. Akkaş, "Use of xylan, an agricultural by-product, in wheat gluten based biodegradable films: Mechanical, solubility and water vapor transfer rate properties," Bioresource Technology, vol. 87, no. 3, pp. 239-246, 2003.
[5] E. I. Goksu, M. Karamanlioglu, U. Bakir, L. Yilmaz, and U. Yilmazer, "Production and characterization of films from cotton stalk xylan," Journal of Agricultural and Food Chemistry, vol. 55, no. 26, pp. 10685-10691, 2007.

[6] X.-W. Peng, J.-L. Ren, L.-X. Zhong, and R.-C. Sun, "Nanocomposite films based on xylan-rich hemicelluloses and cellulose nanofibers with enhanced mechanical properties," Biomacromolecules, vol. 12, no. 9, pp. 3321-3329, 2011.

[7] S. Wang, J. Ren, W. Kong et al., "Influence of urea and glycerol on functional properties of biodegradable PVA/xylan composite films," Cellulose, vol. 21, no. 1, pp. 495-505, 2014.

[8] C.-D. Gao, J.-L. Ren, S.-Y. Wang, R.-C. Sun, and L.-H. Zhao, "Preparation of polyvinyl alcohol/xylan blending films with 1,2,3,4-butane tetracarboxylic acid as a new plasticizer," Journal of Nanomaterials, vol. 2014, Article ID 764031, 8 pages, 2014.

[9] M. Alekhina, K. S. Mikkonen, R. Alén, M. Tenkanen, and H. Sixta, "Carboxymethylation of alkali extracted xylan for preparation of bio-based packaging films," Carbohydrate Polymers, vol. 100, pp. 89-96, 2014.

[10] R. K. Jain, M. Sjöstedt, and W. G. Glasser, "Thermoplastic xylan derivatives with propylene oxide," Cellulose, vol. 7, no. 4, pp. 319336, 2000.

[11] Y. Guan, B. Zhang, X. Tan et al., "Organic-inorganic composite films based on modified hemicelluloses with clay nanoplatelets," ACS Sustainable Chemistry \& Engineering, vol. 2, no. 7, pp. 18111818, 2014.

[12] A. M. Stepan, A. Höije, H. A. Schols, P. De Waard, and P. Gatenholm, "Arabinose content of arabinoxylans contributes to flexibility of acetylated arabinoxylan films," Journal of Applied Polymer Science, vol. 125, no. 3, pp. 2348-2355, 2012.

[13] K. Das, D. Ray, N. R. Bandyopadhyay et al., "Preparation and characterization of cross-linked starch/poly(vinyl alcohol) green films with low moisture absorption," Industrial \& Engineering Chemistry Research, vol. 49, no. 5, pp. 2176-2185, 2010.

[14] A. Abdulkhani, E. Hojati Marvast, A. Ashori, Y. Hamzeh, and A. N. Karimi, "Preparation of cellulose/polyvinyl alcohol biocomposite films using 1-n-butyl-3-methylimidazolium chloride," International Journal of Biological Macromolecules, vol. 62, pp. 379-386, 2013.

[15] Q. Deng, J. Li, J. Yang, and D. Li, “Optical and flexible $\alpha$-chitin nanofibers reinforced poly(vinyl alcohol) (PVA) composite film: fabrication and property," Composites Part A: Applied Science and Manufacturing, vol. 67, pp. 55-60, 2014.

[16] N. T. Nguyen and J. H. Liu, "A green method for in situ synthesis of poly(vinyl alcohol)/chitosan hydrogel thin films with entrapped silver nanoparticles," Journal of the Taiwan Institute of Chemical Engineers, vol. 45, no. 5, pp. 2827-2833, 2014.

[17] D. Song, Y. Zhao, C. Dong, and Y. Deng, "Surface modification of cellulose fibers by starch grafting with crosslinkers," Journal of Applied Polymer Science, vol. 113, no. 5, pp. 3019-3026, 2009.

[18] D. Song, V. Breedveld, and Y. Deng, "Rheological study of self-crosslinking and co-crosslinking of ammonium zirconium carbonate and starch in aqueous solutions," Journal of Applied Polymer Science, vol. 122, no. 2, pp. 1019-1029, 2011.

[19] K. S. Mikkonen, J. Schmidt, A.-H. Vesterinen, and M. Tenkanen, "Crosslinking with ammonium zirconium carbonate improves the formation and properties of spruce galactoglucomannan films," Journal of Materials Science, vol. 48, no. 12, pp. 4205-4213, 2013. 
[20] M. Groendahl, L. Bindgard, L. Eriksson, P. Gatenholm, and T. Hjertberg, "Polymeric film or coating comprising hemicellulose," US Patent no. 0129642, 2010.

[21] B. Ş. Kayserilioğlu, W. M. Stevels, W. J. Mulder, and N. Akkaş, "Mechanical and biochemical characterisation of wheat gluten films as a function of $\mathrm{pH}$ and co-solvent," Starch/Staerke, vol. 53, no. 8, pp. 381-386, 2001.

[22] L. Brandão, L. M. Madeira, and A. M. Mendes, "Mass transport on composite dense PDMS membranes with palladium nanoclusters," Journal of Membrane Science, vol. 288, no. 1-2, pp. 112-122, 2007.

[23] P. Taveira, A. Mendes, and C. Costa, "On the determination of diffusivity and sorption coefficients using different time-lag models," Journal of Membrane Science, vol. 221, no. 1-2, pp. 123133, 2003.

[24] J.-Y. Jo, C.-K. Min, and J.-S. Shin, "Manufacture of waterresistant corrugated board boxes for agricultural products in the cold chain system," Journal of Korea Technical Association of the Pulp and Paper Industry, vol. 44, no. 2, pp. 29-34, 2012.

[25] I. Spiridon, C.-A. Teacă, R. Bodîrlău, and M. Bercea, "Behavior of cellulose reinforced cross-linked starch composite films made with tartaric acid modified starch microparticles," Journal of Polymers and the Environment, vol. 21, no. 2, pp. 431-440, 2013.

[26] L. C. Tomé, C. M. B. Gonalves, M. Boaventura et al., "Preparation and evaluation of the barrier properties of cellophane membranes modified with fatty acids," Carbohydrate Polymers, vol. 83, no. 2, pp. 836-842, 2011.

[27] E. Chiellini, A. Corti, S. D’Antone, and R. Solaro, "Biodegradation of poly (vinyl alcohol) based materials," Progress in Polymer Science, vol. 28, no. 6, pp. 963-1014, 2003. 

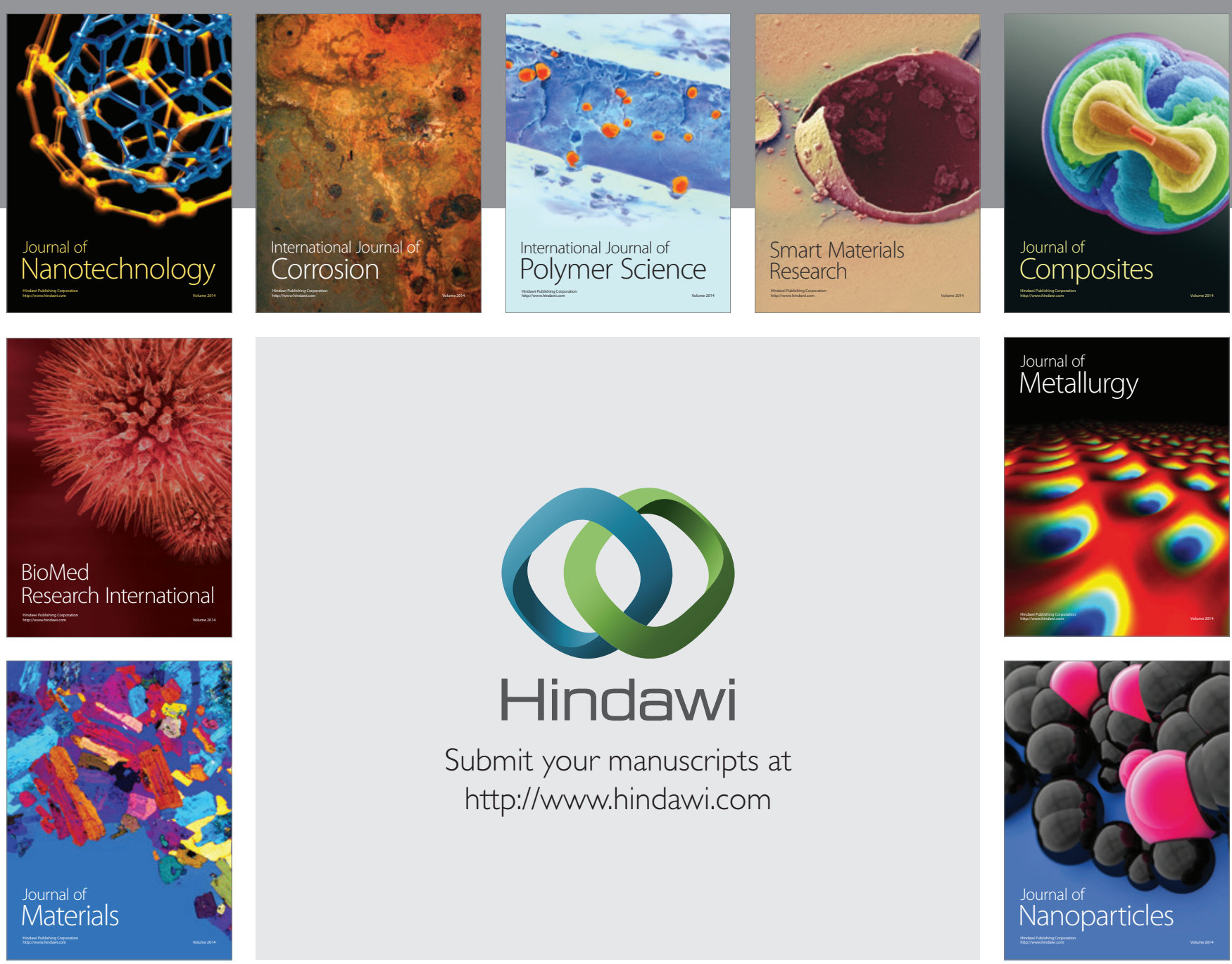

Submit your manuscripts at http://www.hindawi.com
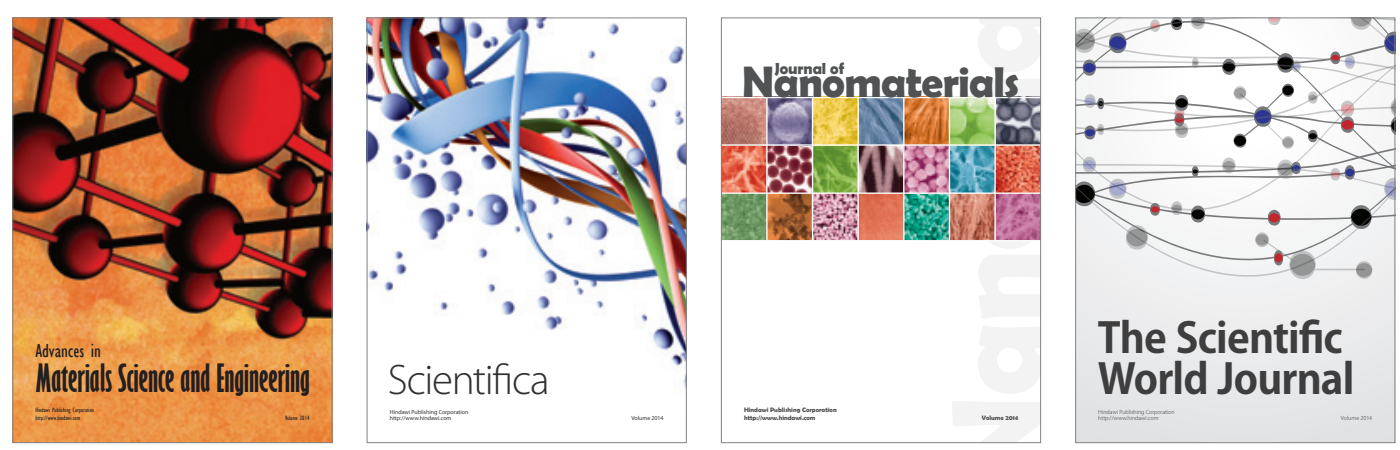

\section{The Scientific World Journal}
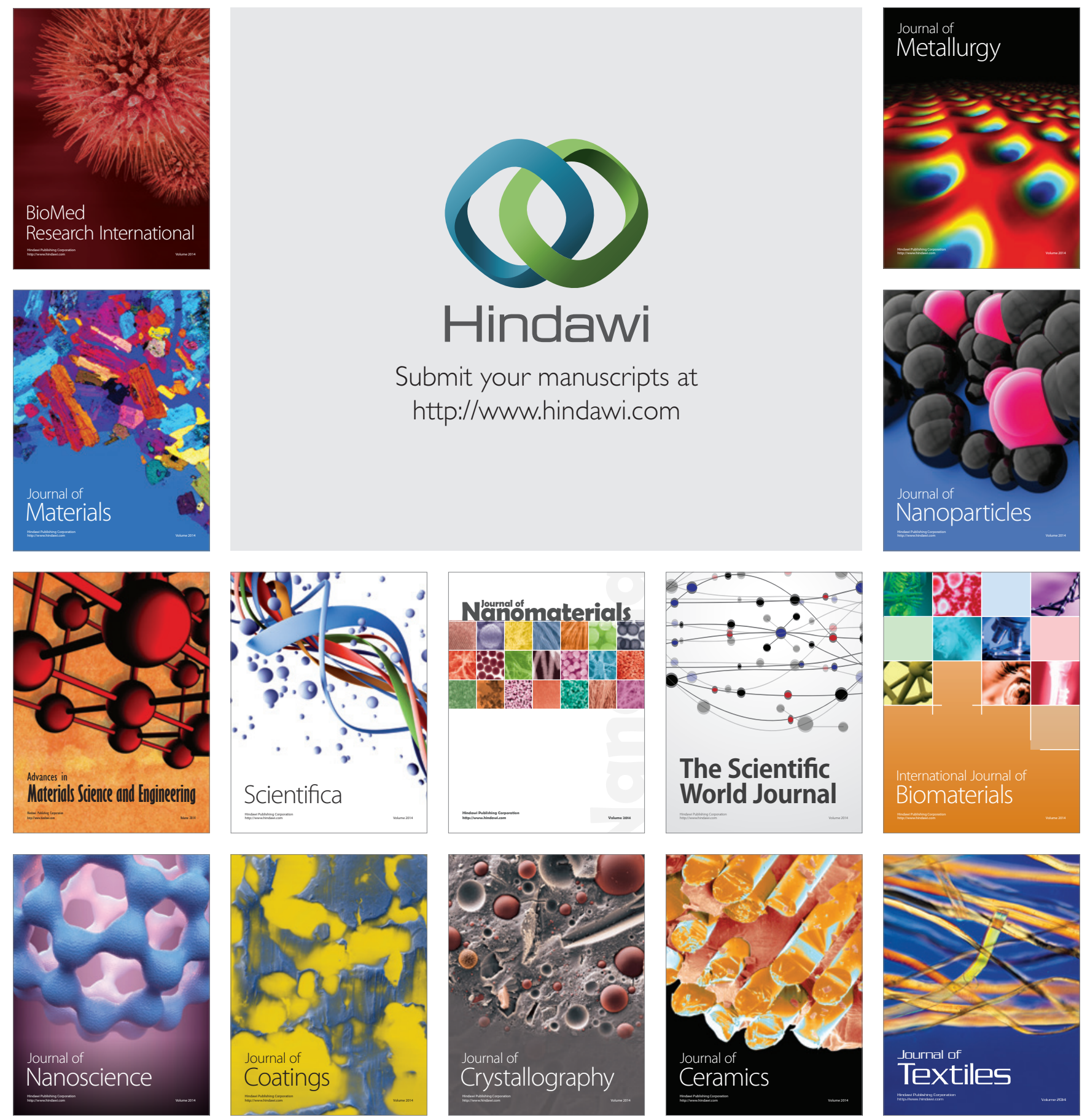